\title{
Tunneling magnetoresistance in diluted magnetic semiconductor tunnel junctions
}

\author{
Pin Lyut and Kyungsun Moon \\ Department of Physics and Institute of Physics and Applied Physics, Yonsei University, Seoul 120-749, Korea
}

(November 21, 2018)

\begin{abstract}
Using the spin-polarized tunneling model and taking into account the basic physics of ferromagnetic semiconductors, we study the temperature dependence of the tunneling magnetoresistance (TMR) in the diluted magnetic semiconductor (DMS) trilayer heterostructure system (Ga,Mn)As/AlAs/(Ga,Mn)As. The experimentally observed TMR ratio is in reasonable agreement with our result based on the typical material parameters. It is also shown that the TMR ratio has a strong dependence on both the itinerantcarrier density and the magnetic ion density in the DMS electrodes. This can provide a potential way to achieve larger TMR ratio by optimally adjusting the material parameters.
\end{abstract}

PACS numbers: 72.25.-b, 73.40.Ty, 73.40.Gk, 75.50.Pp

\section{INTRODUCTION}

Since the early experiment done by Julliere 1 there has been considerable interest in studying the tunneling magnetoresistance (TMR) effect in magnetic tunnel junctions 2 目 due to its promising applications in digital storage and magnetic sensor technology. The conventional electrodes of magnetic tunnel junctions are made up of the ferromagnetic transition metals such as Fe, Co, $\mathrm{Ni}$, and their alloys. Usually the TMR effect in the conventional magnetic tunnel junctions is described by the Julliere model. 1 . The electrons near at the Fermi levels of the electrodes participate in the tunneling process and have the main contribution to the tunneling conductance and so to the TMR effect.

Recent discovery of the ferromagnetism in the diluted magnetic semiconductors (DMS) has opened the rich field of an interesting interplay between magnetic and semiconducting properties, 60 which makes it possible to combine the information processing and data storage in one material. At high concentration $c$ of randomly distributed $\mathrm{Mn}^{2+}$ ions doped in GaAs samples with high hole density $c^{*}, \mathrm{Ga}_{1-x} \mathrm{Mn}_{x}$ As compounds exhibit ferromagnetism with transition temperature as high as $110 \mathrm{~K}$ at certain value of $x$. The origin of ferromagnetism in the DMS can be explained by the itinerant holes through the $p$ - $d$ exchange coupling between the valanceband holes and the spins of impurity $\mathrm{Mn}$ ions $\mathrm{d}$ In order to investigate the spinpolarized electron transport phenomenon in the DMS ferromagnetic semiconductor heterostructures, the epitaxial tunnel junctions have been successfully fabricated in the form of $\mathrm{Ga}_{0.961} \mathrm{Mn}_{0.039} \mathrm{As} / \mathrm{AlAs} / \mathrm{Ga}_{0.961} \mathrm{Mn}_{0.039}$ As. The TMR effect was observed and the maximum TMR ratio thus obtained is as high as $44.4 \%$ at 4.2 K.9 The subsequent experiment 4 also realized the TMR effect in the DMS tunnel junctions of $\mathrm{Ga}_{0.95} \mathrm{Mn}_{0.05} \mathrm{As} / \mathrm{Al}_{y} \mathrm{Ga}_{1-y} \mathrm{As} / \mathrm{Ga}_{0.97} \mathrm{Mn}_{0.03}$ As.

The model1.5 with several necessary extensions $11-15$ is known to describe the tunnel junctions, in which the two electrodes are made of the conventional ferromagnetic metals or the half-metallic manganites such as $\mathrm{La}_{1-x} \mathrm{Sr}_{x} \mathrm{MnO}_{3}$. For the manganite tunnel junctions, various tunneling processes are included in the model as follows. The elastic spin-flip scattering of the spin polarized carriers by the local magnetic impurities within the insulating barrier and the inelastic spin-flip scattering induced by the thermal collective excitations of local spins at the interfaces between the insulating barrier and the manganite electrodes are the two basic ingredients. By taking into account the temperature dependence of the spin polarization of itinerant carriers, one can calculate the temperature dependence of the TMR ratio. 15 In the paper, we have applied the above generalized Julliere model to the DMS tunnel junctions and obtained the various properties of the TMR effects of the system.

The paper is organized as follows. In Sec. II, we propose a spin-polarized tunneling model for the DMS tunnel junctions by generalizing the previous spin-polarized tunneling model in order to correctly take into account the basic physics of the DMS. In Sec. III, we show that the experimentally observed TMR ratio in the DMS tunnel junctions can be well reproduced from our calculations with the appropriate choice of typical material parameters and also show that the TMR ratio thus obtained is strongly dependent on both the itinerant-carrier density and the magnetic ion density in the DMS electrodes. Finally we conclude with a brief summary in Sec.IV.

\section{SPIN-POLARIZED TUNNELING MODEL}

We will briefly summarize the basic physics of ferromagnetism occurring in certain DMS compounds following the seminal work of König et al..B

In the doping range of our interest, that is, $0.035<$ $x<0.06, \mathrm{Ga}_{1-x} \mathrm{Mn}_{x} \mathrm{As}$ can be described as a degenerate semiconductor with relatively high hole concentrations and the $\mathrm{Mn}^{2+}$ ions act as local magnetic impurities.

The Hamiltonian describing the basic physics of the DMS can be written as 


$$
\begin{gathered}
\mathcal{H}=\int d^{3} r \sum_{\sigma} \Psi_{\sigma}^{\dagger}(\mathbf{r})\left(-\frac{\hbar^{2}}{2 m^{*}} \nabla^{2}-\mu^{*}\right) \Psi_{\sigma}(\mathbf{r}) \\
+J_{p d} \int d^{3} r \mathbf{S}(\mathbf{r}) \cdot \mathbf{s}(\mathbf{r}),
\end{gathered}
$$

where $\mathbf{S}(\mathbf{r})$ stands for the spin density of the local magnetic impurities, which is given by $\sum_{I} \mathbf{S}_{I} \delta\left(\mathbf{r}-\mathbf{R}_{I}\right)$ with $\mathbf{R}_{I}$ denoting the positions of magnetic impurities. The field operator $\Psi_{\sigma}^{\dagger}(\mathbf{r})\left(\Psi_{\sigma}(\mathbf{r})\right)$ is the creation (annihilation) operator for the itinerant hole at position $\mathbf{r}$ with spin $\sigma$. The $\mathbf{s}(\mathbf{r})$ represents the spin density of itinerantcarriers, which can be written in terms of field operators as $\frac{1}{2} \sum_{\sigma \sigma^{\prime}} \Psi_{\sigma}^{\dagger}(\mathbf{r}) \vec{\tau}_{\sigma \sigma^{\prime}} \Psi_{\sigma^{\prime}}(\mathbf{r})$, where $\vec{\tau}$ are the three Pauli spin matrices. The $m^{*}$ and $\mu^{*}$ are the effective mass and chemical potential of itinerant carriers, respectively. The $J_{p d}$ stands for the $p$ - $d$ exchange coupling strength between itinerant holes and $\mathrm{Mn}^{2+}$ ion impurity spins. The recent experiments suggest that the itinerant hole density $c^{*}$ is much lower than the magnetic ion density $c$ satisfying $c^{*} / c \ll 1$. Here we focus our attention on the DMS with ferromagnetic ground state. Using the Holstein-Primakoff transformations and performing the coarse graining procedures, the local spin density $\mathbf{S}(\mathbf{r})$ can be written in terms of the newly defined bosonic fields $b^{\dagger}(\mathbf{r}), b(\mathbf{r})$ as follows

$$
\begin{gathered}
S^{+}(\mathbf{r})=\sqrt{2 c S-b^{\dagger}(\mathbf{r}) b(\mathbf{r})} b(\mathbf{r}) \\
S^{-}(\mathbf{r})=b^{\dagger}(\mathbf{r}) \sqrt{2 c S-b^{\dagger}(\mathbf{r}) b(\mathbf{r})} \\
S^{z}(\mathbf{r})=c S-b^{\dagger}(\mathbf{r}) b(\mathbf{r})
\end{gathered}
$$

The self-consistent spin wave approximation developed in Ref. 8 yields the following effective Hamiltonian for the DMS system

$$
\mathcal{H}_{\mathrm{eff}}=\sum_{\mathbf{k} \sigma} \varepsilon_{\mathbf{k} \sigma} c_{\mathbf{k} \sigma}^{\dagger} c_{\mathbf{k} \sigma}+\sum_{|\mathbf{q}|<q_{c}} \Omega_{\mathbf{q}} b_{\mathbf{q}}^{\dagger} b_{\mathbf{q}},
$$

where $\varepsilon_{\mathbf{k} \sigma}$ stands for the band energy of the itinerant holes with spin $\sigma= \pm 1$, which is given by

$$
\varepsilon_{\mathbf{k} \sigma}=\varepsilon_{\mathbf{k}}+\frac{\Delta}{2} \sigma
$$

and $\varepsilon_{\mathbf{k}}=\hbar^{2} k^{2} /\left(2 m^{*}\right)$. The spin wave dispersion $\Omega_{\mathbf{q}}$ is given by

$$
\begin{aligned}
\Omega_{\mathbf{q}}=\frac{J_{p d}}{2}\left(n_{\downarrow}\right. & \left.-n_{\uparrow}\right)-\frac{J_{p d} \Delta}{2 V} \sum_{\mathbf{k}}\left[f\left(\varepsilon_{\mathbf{k} \downarrow}\right)-f\left(\varepsilon_{\mathbf{k}+\mathbf{q} \uparrow}\right)\right] \\
& \times \frac{1}{\Omega_{\mathbf{q}}+\varepsilon_{\mathbf{k}+\mathbf{q} \uparrow}-\varepsilon_{\mathbf{k} \downarrow}},
\end{aligned}
$$

where $c_{\mathbf{k}}$ and $b_{\mathbf{q}}$ are the itinerant hole and the spin wave annihilation operators in the momentum space respectively, and $f(x)$ is the Fermi-Dirac distribution function. Here $n_{\downarrow}\left(n_{\uparrow}\right)$ is the spin-down (spin-up) itinerant hole density satisfying $n_{\uparrow}+n_{\downarrow}=c^{*}$, the exchange gap $\Delta=c J_{p d} S$, and $q_{c}$ is the Debye cutoff for the spin waves of local impurity spins with $q_{c}^{3}=6 \pi^{2} c$. At finite temperatures, the spin wave dispersion can be generalized by imposing the following self-consistency conditions for the finite temperature exchange gap : $\Delta(T)=J_{p d}\left\langle S^{z}\right\rangle$. Here $\left\langle S^{z}\right\rangle$ represents the thermal average of the impurity Mn ion spins, which are approximately calculated by the following formula

$$
\begin{gathered}
\left\langle S^{z}\right\rangle=\frac{1}{V} \sum_{|\mathbf{q}|<q_{c}} S \mathcal{B}_{s}\left(\beta S \Omega_{\mathbf{q}}\right) \\
=\frac{1}{V} \sum_{|\mathbf{q}|<q_{c}}\left\{S-n_{B}\left(\Omega_{\mathbf{q}}\right)+(2 S+1) n_{B}\left[(2 S+1) \Omega_{\mathbf{q}}\right]\right\},
\end{gathered}
$$

where $\mathcal{B}_{s}(x)$ is the Brillouin function, $n_{B}(x)$ the BoseEinstein distribution function, and $\beta=1 / k_{B} T$. The second term in the second equality of Eq. (8) describes how the thermally induced spin waves from the independent Bose statistics with no limit in the number of spin waves indeed reduce the magnetization of the system. The third term takes into account the correct spin kinematics, which rules out the unphysical states.

Based on the above scheme, one can calculate the magnetization of the Mn impurity spins and the band structure of the itinerant holes approximately self-consistently by solving a set of coupled equations. In this theoretical framework, the non-monotonic dependence of the Curie temperature on the itinerant hole density $c^{*}$ has been successfully obtained.

Now we consider the DMS magnetic tunnel junctions in which both electrodes are made of the ferromagnetic DMS $\mathrm{Ga}_{1-x} \mathrm{Mn}_{x}$ As compounds. The carrier tunneling through a thin AlAs insulating barrier involves removing a carrier at one side and creating it at the other side with spin conserving or spin-flipping. The spin-flip tunneling processes can be divided into two different categories: The elastic spin-flip tunneling arising from the $\mathrm{Mn}^{2+}$ ion impurity spins located within the insulating barrier and the inelastic spin-flip tunneling induced by the thermal collective excitations of the impurity spins near at the interfaces between the insulating barrier and the DMS electrodes.

For the collinear magnetization configurations, the tunneling Hamiltonian of the DMS tunnel junctions can be written as

$$
\begin{gathered}
H=H_{\mathrm{L}}+H_{\mathrm{R}}+H_{\mathrm{T}}, \\
H_{\mathrm{L}}=\sum_{\mathbf{p} \sigma} \varepsilon_{\mathbf{p} \sigma} c_{\mathbf{p} \sigma}^{\dagger} c_{\mathbf{p} \sigma}+\sum_{|\mathbf{q}|<q_{c}} \Omega_{\mathbf{q}} b_{\mathbf{q} L}^{\dagger} b_{\mathbf{q}, L},
\end{gathered}
$$




$$
\begin{gathered}
H_{\mathrm{R}}=\sum_{\mathbf{k} \sigma} \varepsilon_{\mathbf{k} \sigma} d_{\mathbf{k} \sigma}^{\dagger} d_{\mathbf{k} \sigma}+\sum_{|\mathbf{q}|<q_{c}} \Omega_{\mathbf{q}} b_{\mathbf{q} R}^{\dagger} b_{\mathbf{q} R}, \\
H_{\mathrm{T}}=\sum_{\mathbf{k} \mathbf{p} \sigma}\left(T_{\mathbf{k p}}^{0} d_{\mathbf{k} \sigma}^{\dagger} c_{\mathbf{p} \sigma}+i T_{\mathbf{k} \mathbf{p}}^{\prime} d_{\mathbf{k} \sigma}^{\dagger} c_{\mathbf{p},-\sigma}+\text { H.c. }\right) \\
+\frac{1}{\sqrt{V}} \sum_{\mathbf{k p},|\mathbf{q}|<q_{c}}\left\{T_{\mathbf{k p q}}^{\prime \prime}\left[S_{L}^{z}(\mathbf{q})+S_{R}^{z}(\mathbf{q})\right]\left(d_{\mathbf{k} \uparrow}^{\dagger} c_{\mathbf{p} \uparrow}-d_{\mathbf{k} \downarrow}^{\dagger} c_{\mathbf{p} \downarrow}\right)\right. \\
+ \text { H.c. }\}+\frac{1}{\sqrt{V}} \sum_{\mathbf{k p},|\mathbf{q}|<q_{c}}\left\{T_{\mathbf{k p q}}^{\prime \prime}\left[\sqrt{2 c S_{L}} b_{\mathbf{q} L}+\sqrt{2 c S_{R}} b_{\mathbf{q} R}\right]\right. \\
\left.\times\left(d_{\mathbf{k} \downarrow}^{\dagger} c_{\mathbf{p} \uparrow}+c_{\mathbf{p} \downarrow}^{\dagger} d_{\mathbf{k} \uparrow}\right)+\text { H.c. }\right\} .
\end{gathered}
$$

Here $H_{\mathrm{L}}\left(H_{\mathrm{R}}\right)$ is the effective Hamiltonian for the bulk DMS in the left (right) electrode, which has been derived from the original Hamiltonian of Eq. (1) as explained earlier. The operator $d_{\mathbf{k} \sigma}\left(c_{\mathbf{p} \sigma}\right)$ is the carrier annihilation operator with momentum $\mathbf{k}(\mathbf{p})$ and spin $\sigma$ of the right (left) DMS electrode, and $S_{\alpha}^{z}(\mathbf{q})=c S_{\alpha}-b_{\mathbf{q} \alpha}^{\dagger} b_{\mathbf{q} \alpha}$ with $\alpha=L, R$.

The term with coefficient $T_{\mathbf{k p}}^{\prime}$ in $H_{\mathrm{T}}$ represents the spin-flip tunneling arising from the $\mathrm{Mn}^{2+}$ ion impurity spins inside the AlAs insulating barrier. This type of spin-flip tunneling has been introduced to the tunneling Hamiltonian for the conventional ferromagnetic tynnel junctions 12 and the manganite tunnel junctions. 14.5 The imaginary $i$ for the spin-flip tunneling matrix elements indicates that the elastic spin-flip tunneling processes are incoherent in any magnetization configurations. As a generalization of the typical tunneling Hamiltonian 16 to the DMS tunnel junctions, the last two terms have been included in the tunneling Hamiltonian of Eq. (12), which describe the inelastic spin-flip tunneling process induced by the thermal spin wave collective excitations of local spins near at the interfaces between the insulating barrier and the DMS electrodes. Since this kind of carrier tunneling involves the emission or absorption of the local spin collective excitations near the left or right interfaces, it is an inelastic tunneling process. The inelastic spin-flip tunneling may occur not only directly at the interfaces but also within the coherence length of the interfaces, which is relatively long comparing to the barrier width. It then allows us to use the bulk spin wave dispersion relation in the tunneling Hamiltonian. The fact that the wave vector $\mathbf{k}$ is independent of $\mathbf{p}$ in Eq. (12) implies that we only consider the incoherent tunneling processes, which are known to be appropriate for the defect-populated insulating barriers and the rough interfaces. As usual, we make the following assumptions that the strengths of various tunneling amplitudes, that is, $\left|T_{\mathbf{k p}}^{0}\right|^{2},\left|T_{\mathbf{k p}}^{\prime}\right|^{2}$, and $\left|T_{\mathbf{k p q}}^{\prime \prime}\right|^{2}$ are independent of momenta $\mathbf{k}, \mathbf{p}, \mathbf{q}$ and then will be taken to be their average values $\left|T^{0}\right|^{2},\left|T^{\prime}\right|^{2}$, and
$\left|T^{\prime \prime}\right|^{2}$, respectively. By making a transformation for the tunneling Hamiltonian from the collinear magnetization configurations to arbitrary relative angle $\theta$ between the spin orientations of the two electroges 17 and using the standard Green's function methods, 18 one can obtain the tunneling conductance $G(\theta)$ at zero bias up to the leading order in the tunneling amplitudes, which is given by

$$
\begin{gathered}
G(\theta)=\frac{\pi e^{2}}{\hbar}\left|T^{0}\right|^{2}\left[\rho_{\downarrow}\left(\varepsilon_{F}\right)+\rho_{\uparrow}\left(\varepsilon_{F}\right)\right]^{2}\left[1+P^{2} \cos \theta\right. \\
\left.+\gamma\left(1-P^{2} \cos \theta\right)\right],
\end{gathered}
$$

where $P$ stands for the itinerant carrier spin polarization given by

$$
P=\frac{\rho_{\downarrow}\left(\varepsilon_{F}\right)-\rho_{\uparrow}\left(\varepsilon_{F}\right)}{\rho_{\downarrow}\left(\varepsilon_{F}\right)+\rho_{\uparrow}\left(\varepsilon_{F}\right)},
$$

and $\rho_{\downarrow}\left(\varepsilon_{F}\right)\left(\rho_{\uparrow}\left(\varepsilon_{F}\right)\right)$ is the density of states (DOS) of the spin-down (spin-up) carriers at the Fermi level of the DMS electrodes. The relative angle $\theta$ may be tunable by applying a magnetic field and $\gamma$ is given by the following relation

$$
\gamma=\gamma_{1}+4 \eta c S\left(c S-\left\langle S^{z}\right\rangle\right),
$$

where $\gamma_{1}=\left|T^{\prime}\right|^{2} /|\widetilde{T}|^{2}$ and $\eta=\left|T^{\prime \prime}\right|^{2} /|\widetilde{T}|^{2}$ with $|\widetilde{T}|^{2}=$ $\left|T^{0}\right|^{2}+2 c^{2} S^{2}\left|T^{\prime \prime}\right|^{2} \simeq\left|T^{0}\right|^{2}$, since $c^{2}\left|T^{\prime \prime}\right|^{2}$ is usually about two orders of magnitude smaller than $\left|T^{0}\right|^{2} .16 \mathrm{In}$ deriving Eq. (15), we have used the following relation $V^{-1} \sum_{|\mathbf{q}|<q_{c}}\left\langle b_{\mathbf{q}}^{\dagger} b_{\mathbf{q}}\right\rangle=c S-\left\langle S^{z}\right\rangle$. The corresponding tunneling resistance is given by $R(\theta)=1 / G(\theta)$.

The TMR ratio is defined by the following formula

$$
\left(\frac{\Delta R}{R_{\mathrm{P}}}\right)_{\max }=\frac{R_{\mathrm{AP}}-R_{\mathrm{P}}}{R_{\mathrm{P}}},
$$

where $R_{\mathrm{AP}}$ and $R_{\mathrm{P}}$ represent the tunneling resistance in the antiparallel and parallel spin alignments of the two ferromagnetic electrodes respectively, and the maximum TMR ratio for the DMS tunnel junctions can be obtained as

$$
\left(\frac{\Delta R}{R_{\mathrm{P}}}\right)_{\max }=\frac{2(1-\gamma) P^{2}}{1-P^{2}+\gamma\left(1+P^{2}\right)} .
$$

If the electrodes of the DMS tunnel junctions are made of the non-degenerate DMSs, the tunneling conductance will be proportional to the itinerant carrier density and the TMR ratio will be more appropriately given by Eq. (17) with the carrier spin polarization replaced by $P=\left(n_{\downarrow}-n_{\uparrow}\right) /\left(n_{\downarrow}+n_{\uparrow}\right)$. The different definitions of the carrier spin polarization in the TMR ratio for the degenerate and non-degenerate DMS tunnel junctions are due to the distinctly different transport properties in the corresponding DMSs. 


\section{RESULTS AND DISCUSSIONS}

In Fig. 1 and Fig. 2 are illustrated the temperature dependences of the impurity $\mathrm{Mn}$ ion magnetization of the DMS, the itinerant-carrier spin polarization, and the maximum TMR ratio for the DMS tunnel junctions at several different itinerant-carrier densities $c^{*}$ with the following choice of material parameters: $\gamma_{1}=0.692$, $\eta=0.005 \mathrm{~nm}^{6}$, and $m^{*}=0.5 m_{e}, J_{p d}=0.15 \mathrm{eVnm}^{3}$ appropriate for the bulk $\mathrm{Ga}_{1-x} \mathrm{Mn}_{x} \mathrm{As}$ compounds 6 and fixed impurity $\mathrm{Mn}$ ion density $c=1 \mathrm{~nm}^{-3}$, where $m_{e}$ is the free electron mass. Figure 1 (a) shows the impurity Mn ion magnetization $\left\langle S^{z}\right\rangle$ normalized by $c$. One can notice that the critical temperature $T_{c}$ decreases with the further increase of the itinerant carrier density $c^{*}$ from $c^{*}=0.1 \mathrm{~nm}^{-3}$. From the solid curves of Fig. 1 (b) corresponding to $c^{*}=0.1 \mathrm{~nm}^{-3}$, one can see the following features that at relatively low temperatures, the spin-up carrier band is completely lifted from the spin-down carrier band by the large exchange gap $\Delta(T)$. At the meanfield level, this leads to the complete spin polarization of the itinerant carriers, i.e. $P=100 \%$, exhibiting the halfmetallic feature. In this case the TMR ratio is reduced to $\left(\Delta R / R_{p}\right)_{\max }=1 / \gamma-1$, which decreases with the increase in the population of the thermally excited spin wave collective excitations leading to the increase of $\gamma$ as shown in Fig. 1 (c). As the temperature increases, the size of the exchange gap $\Delta(T)$ is gradually reduced. With the further increase of temperature beyond a certain value, the number of the majority spin-down carriers begins to decrease, while that of the minority spin-up carriers starts to increase. At high temperatures, the carrier spin polarization decreases rapidly with temperatures, which makes the TMR effect disappear above a critical temperature. The factor of $(1-\gamma)$ in Eq. (17) indicates that the TMR effect may vanish below the critical temperature, which is due to the competition between the spin-conserving tunneling amplitude $\left|T^{0}\right|^{2}$ and the spin-flip tunneling ones $\left|T^{\prime}\right|^{2},\left|T^{\prime \prime}\right|^{2}$.

For higher carrier densities, say, $c^{*}=0.2 \mathrm{~nm}^{-3}$, the Fermi energy of the system increases and then both spin states are occupied losing the half-metallic feature as shown in Fig. 1 (b). Since the partial carrier spin polarization is not beneficial to the TMR effect, it leads to the low TMR ratio. Figure 2 (a) illustrates the impurity Mn ion magnetization $\left\langle S^{z}\right\rangle$ normalized by $c$ at different values of $c^{*}$. In contrary to Fig. 1 (a), one can see that $T_{c}$ decreases with the decrease of the itinerant carrier density $c^{*}$ from $c^{*}=0.05 \mathrm{~nm}^{-3}$ exhibiting the non-monotonic dependence of $T_{c}$ on $c^{*}$, which has been emphasized in Ref. 8. For $c=1 \mathrm{~nm}^{-3}$, the optimal carrier densities, which will achieve the maximum TMR ratio, are shown to be in the range of $0.05 \mathrm{~nm}^{-3}<c^{*}<0.1 \mathrm{~nm}^{-3}$.

Recently Hayashi et al. have performed an experiment for $\mathrm{Ga}_{0.961} \mathrm{Mn}_{0.039} \mathrm{As} / \mathrm{AlAs} / \mathrm{Ga}_{0.961} \mathrm{Mn}_{0.039} \mathrm{As}$ tunnel junctions and obtained the TMR ratio of about $44 \%$ at $4.2 \mathrm{~K} . \mathrm{B}$ By fitting the above experimental data to our calculations, we were able to estimate the approximate values of material parameters: $c^{*}=0.13 \mathrm{~nm}^{-3}$, $c=0.86 \mathrm{~nm}^{-3}$, and $\gamma_{1}=0.692$. The above value of carrier density $c^{*}$ is about $15 \%$ of the $\mathrm{Mn}$ ion concentration $c$. The value of $\gamma_{1}$ indicates that there exists strong spinflip scattering by the Mn ions within the insulating barrier. During the fabrication process of the DMS tunnel junctions, the highly doped $\mathrm{Mn}$ ions in the $\mathrm{Ga}_{1-x} \mathrm{Mn}_{x} \mathrm{As}$ layers can easily diffuse into the AlAs insulating layer. Reducing the Mn ions in the insulating layer is crucial to achieve a large TMR ratio in the DMS tunnel junctions.

Figure 3 shows the temperature dependences of the impurity Mn ion magnetization of the DMS, the itinerantcarrier spin polarization, and the maximum TMR ratio for the DMS tunnel junctions at several values of impurity Mn ion densities $c$ with fixed carrier density $c^{*}=0.1 \mathrm{~nm}^{-3}$. Figures $3(\mathrm{a})$ and (b) demonstrate that the carrier band structure is mainly determined from the size of the exchange gap $\Delta(T)$. At low impurity densities, say, $c=0.6 \mathrm{~nm}^{-3}$, the strength of the ferromagnetic coupling is weak, which induces partial carrier spin polarization. By increasing $c$, the ferromagnetic coupling becomes strong, which completely polarizes the carrier spins above a certain value of $c$. So achieving the high doping of the impurity Mn ions is necessary to have much higher $T_{c}$ and larger TMR ratio at high temperatures.

When the magnetic ion density is beyond a certain large value, say, $c=1.5 \mathrm{~nm}^{-3}$, the impurity ion magnetization and the itinerant-carrier spin polarization seem to exhibit the behaviors of the first order ferromagnetic transition as shown in Fig. 3 (a) and (b). In this case, the first order ferromagnetic transition is induced by the $p$ - $d$ exchange coupling, in contrast to that for the twodimensional quantum well system at very low carrier densities, which is argued to be due to the carrier-carrier correlation effects 19

Our results clearly demonstrate that the TMR effects in the DMS tunnel junctions are dominated by both the ferromagnetic coupling strength and the carrier band structure through the impurity ion density and the itinerant carrier density in the DMS electrodes. The elastic and inelastic spin-flip tunnelings play an important role in reducing the TMR ratio.

\section{SUMMARY}

In summary, we have presented the TMR formula for the DMS tunnel junctions by generalizing the previous spin-polarized tunneling model and taking into account the basic physics of the DMS. The experimentally observed TMR ratio was reproduced by the appropriate choice of the typical material parameters. It was also shown that the TMR ratio has a strong dependence on the itinerant-carrier density and the magnetic ion density in the DMS electrodes as well. In view of the recent predictions of the room temperature ferromagnetic 
semiconductors the DMS tunnel junctions can be practically very useful in the future digital storage and magnetic sensor technology. Our analysis of the TMR ratio can be potentially useful to achieve larger TMR ratio by optimally adjusting the material parameters.

\section{ACKNOWLEDGMENTS}

This work was supported by the Brain Korea 21 Project and also by Grant No. 1999-2-112-001-5 from the interdisciplinary Research program of the KOSEF.

* Also at Department of Physics and Center for Theoretical Physics, Jilin University, Changchun 130023, China.

${ }^{1}$ M. Julliere, Phys. Lett. A 54, 225 (1975).

2 J. M. De Teresa, A. Barthélémy, A. Fert, J. P. Contour, F. Montaigne, and P. Seneor, Science 286, 507 (1999).

${ }^{3}$ M. R. McCartney, R. E. Dunin-Borkowski, M. R. Scheinfein, D. J. Smith, S. Gider, and S. S. P. Parkin, Science 286, 1337 (1999).

${ }^{4}$ G. A. Prinz, Science 282, 1660 (1998).

${ }^{5}$ S. Maekawa and U. Gäfvert, IEEE Trans. Magn. MAG18, 707 (1982).

${ }^{6}$ H. Ohno, Science 281, 951 (1998); J. Magn. Magn. Mater. 200, 110 (1999).

7 T. Dietl, H. Ohno, F. Matsukura, J. Cibert, and D. Ferrand, Science 287, 1019 (2000).

${ }^{8}$ J. König, H.-H. Lin, and A. H. MacDonald, Phys. Rev. Lett. 84, 5628 (2000).

${ }^{9}$ T. Hayashi, H. Shimada, H. Shimizu, and M. Tanaka, J. Cryst. Growth 201/202, 689 (1999).

${ }^{10}$ D. Chiba, N. Akiba, F. Matsukura, Y. Ohno, and H. Ohno, Appl. Phys. Lett. 77, 1873 (2000).

11 J. C. Slonczewski, Phys. Rev. B 39, 6995 (1989).

${ }^{12}$ R. Y. Gu, D. Y. Xing, and J. Dong, J. Appl. Phys. 80, 7163 (1996).

${ }^{13}$ F. Guinea, Phys. Rev. B 58, 9212 (1998).

${ }^{14}$ P. Lyu, D. Y. Xing, and J. Dong, Phys. Rev. B 58, 54 (1998).

${ }^{15}$ P. Lyu, D. Y. Xing, and J. Dong, Phys. Rev. B 60, 4235 (1999).

16 S. Zhang, P. M. Levy, A. C. Marley, and S. S. P. Parkin, Phys. Rev. Lett. 79, 3744 (1997).

${ }^{17}$ P. Lyu, D. Y. Xing, and J. Dong, J. Magn. Magn. Mater. 202, 405 (1999).

18 G. D. Mahan, Many-particle Physics (Plenum, New York, 1981), p.798.

${ }^{19}$ L. Brey and F. Guinea, Phys. Rev. Lett. 85, 2384 (2000). (a)
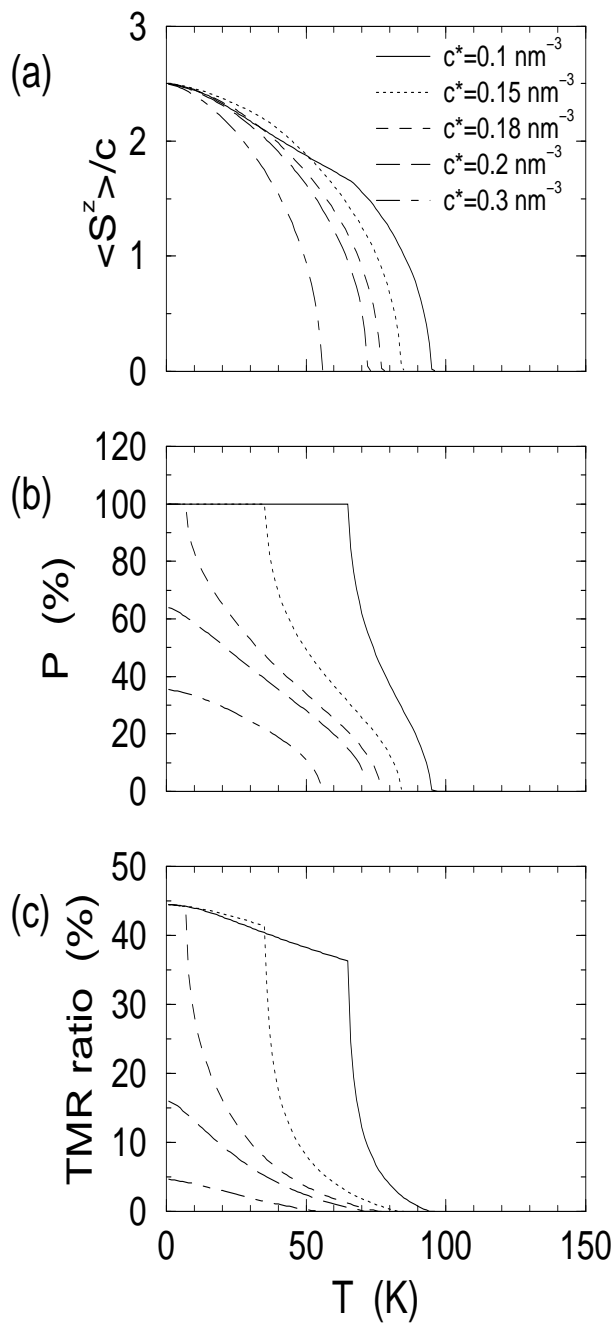

Fig. 1. (a) Impurity Mn ion magnetization of the DMS, (b) itinerant-carrier spin polarization $P$, and (c) the maximum TMR ratio in the DMS tunnel junctions as a function of temperature for several different itinerant-carrier densities $c^{*}=0.3,0.2,0.18,0.15,0.1 \mathrm{~nm}^{-3}$ with the following choice of material parameters $m^{*}=0.5 m_{e}$, $J_{p d}=0.15 \mathrm{eV} \mathrm{nm}^{3}, \gamma_{1}=0.692$ and $\eta=0.005 \mathrm{~nm}^{6}$, 
and the fixed value of $c=1 \mathrm{~nm}^{-3}$.

(a)
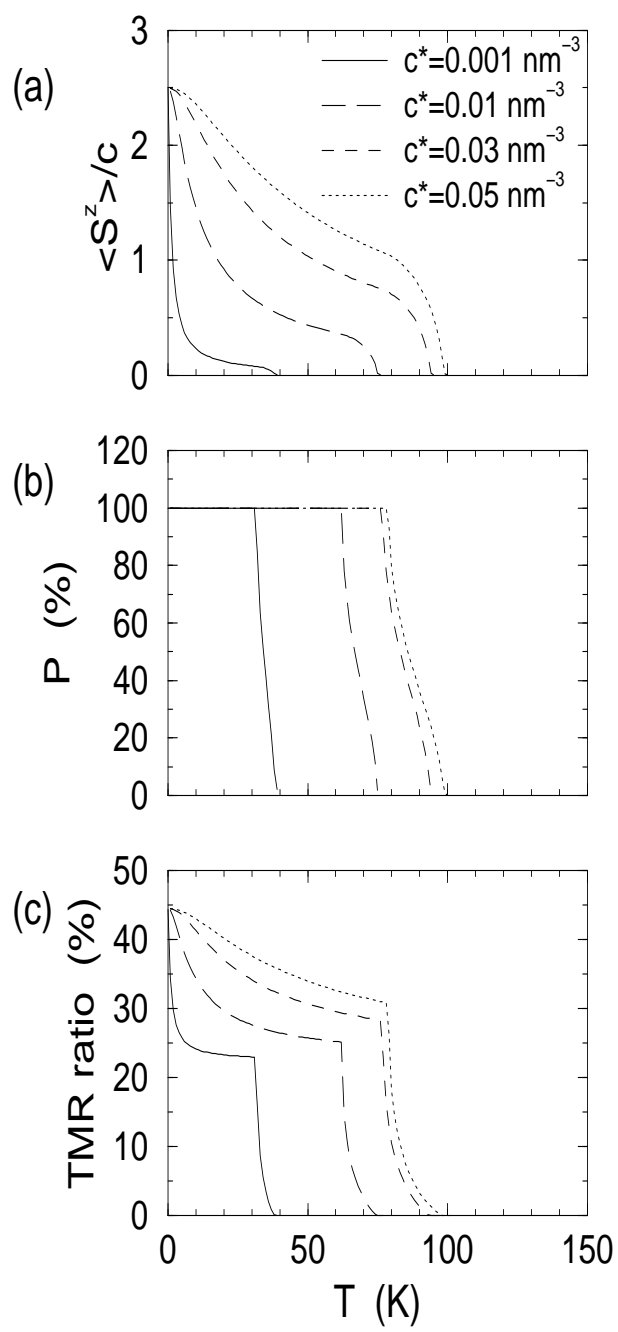

Fig. 2. (a) Impurity Mn ion magnetization of the DMS, (b) itinerant-carrier spin polarization $P$, and (c) the maximum TMR ratio in the DMS tunnel junctions as a function of temperature at several different itinerant-carrier densities $c^{*}=0.05,0.03,0.01,0.001 \mathrm{~nm}^{-3}$ with the following choice of material parameters $m^{*}=0.5 m_{e}$, $J_{p d}=0.15 \mathrm{eV} \mathrm{nm}^{3}, \gamma_{1}=0.692$ and $\eta=0.005 \mathrm{~nm}^{6}$, and the fixed value of $c=1 \mathrm{~nm}^{-3}$.

(a)
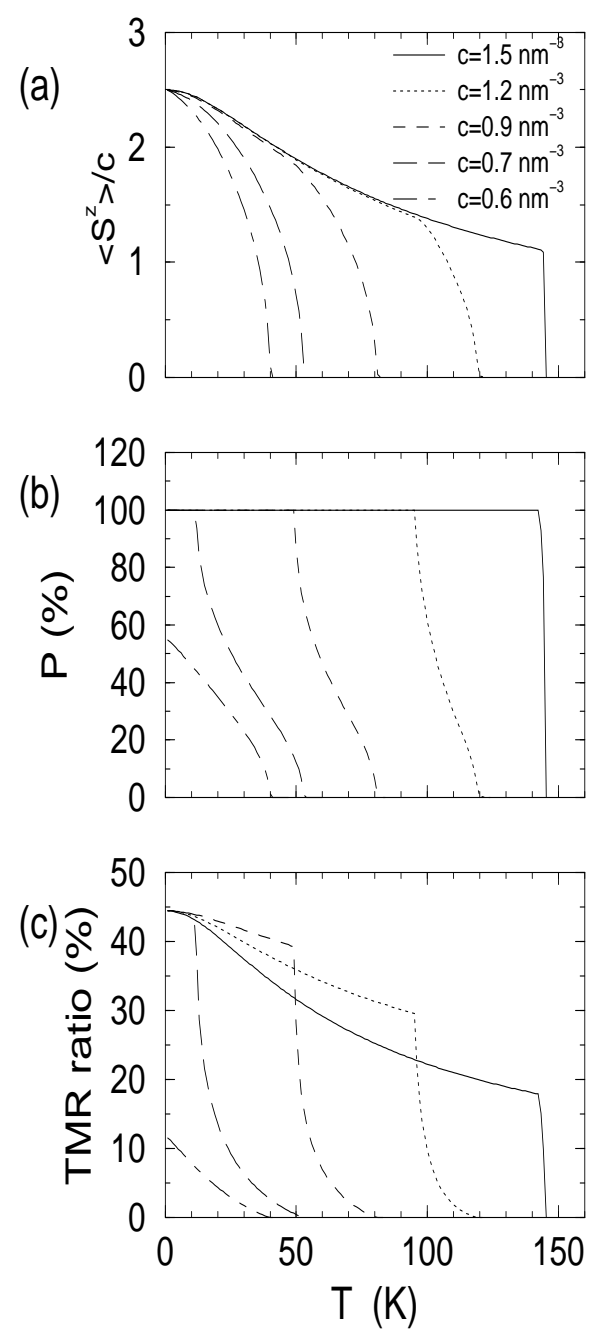

Fig. 3. (a) Impurity Mn ion magnetization of the DMS, (b) itinerant-carrier spin polarization $P$, and (c) the maximum TMR ratio in the DMS tunnel junctions as a function of temperature for several different impurity $\mathrm{Mn}$ ion densities $c=0.6,0.7,0.9,1.2,1.5 \mathrm{~nm}^{-3}$ with the following choice of material parameters $m^{*}=0.5 m_{e}$, $J_{p d}=0.15 \mathrm{eV} \mathrm{nm}^{3}, \gamma_{1}=0.692$ and $\eta=0.005 \mathrm{~nm}^{6}$, and the fixed value of $c^{*}=0.1 \mathrm{~nm}^{-3}$. 